
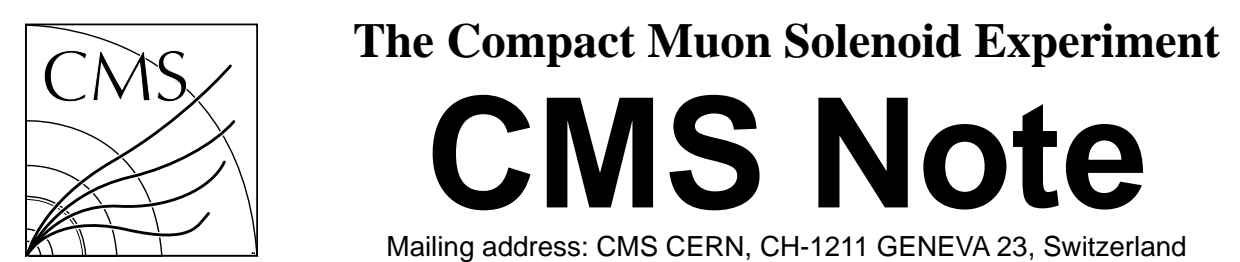

Mailing address: CMS CERN, CH-1211 GENEVA 23, Switzerland

\title{
Search for Charged Higgs in Top Decays at CMS
}

\author{
S. Banerjee, M. Maity \\ Tata Institute of Fundamental Research, Mumbai.
}

\begin{abstract}
We look for light $\mathrm{H}^{ \pm}$produced in top decays by identifying $\tau$-jets from their decays. Significant excess of $t \bar{t}$ events with $\tau$-jets is seen in most of the parameter space explored. An important confirmation of the signal comes from a large deficit of dilepton events in the $t \bar{t}$ sample. According to this study $\mathrm{H}^{ \pm}$with mass upto $160 \mathrm{GeV} / \mathrm{c}^{2}$ may be observed and $170 \mathrm{GeV} / \mathrm{c}^{2}$ may be excluded with low luminosity data from LHC.
\end{abstract}




\section{Introduction}

The Standard Model(SM) of particle physics is in excellent agreement with experimental data. Higgs mechanism is an important part of this model and the physical Higgs boson is yet to be directly observed. As we know, the SM leaves quite a few important issues unresolved. The minimal supersymmetric extension of the SM(MSSM) is a popular theory which attempts to provide solutions to those issues. In MSSM(and many theories beyond the $\mathrm{SM}$ ) we have two Higgs doublets and $\tan \beta$ is the ratio of the vacuum expectation values of the two Higgs doublets. After electroweak symmetry breaking we have five physical Higgs scalars: $h, H\left(M_{h}<M_{H}\right), A$ and $H^{ \pm}$.

Search for $\mathrm{H}^{ \pm}$has been performed at $\operatorname{LEP}$ (pair production)[1] and Tevatron(in decays of top quarks)[2]. LEP experiments have, at 95\% CL, excluded $\mathrm{M}_{\mathrm{H}^{+}}<70 \mathrm{GeV} / \mathrm{c}^{2}$ independent of the value of $\mathrm{BR}\left(\mathrm{H}^{+} \rightarrow \tau^{+} \nu\right)^{1)}$. Similarly, Tevatron experiments have excluded even higher values of $\mathrm{M}_{\mathrm{H}^{+}}$but for limited ranges of $\tan \beta$ (see figure 1). LEP is going to take some more data and RunII of Tevatron is to begin in near future which will explore some more regions in the parameter space.

Still large regions of parameter space for $\mathrm{M}_{\mathrm{H}^{+}}<\mathrm{M}_{t}$ has to be exhaustively searched. In this analysis we search for $\mathrm{H}^{ \pm}$, lighter than than the top quark, produced at $\mathrm{LHC}$ in top quark decays $\left(\mathrm{t} \rightarrow \mathrm{bH}^{+}\right)$where the $\mathrm{H}^{+}$decays into $\tau^{+} \nu$. Discovery of $\mathrm{H}^{ \pm}$will be an unambiguous signal of an extended Higgs sector and possibly of Supersymmetry. Search strategy for $\mathrm{H}^{ \pm}$with the CMS detector has been studied[3] in the context of CMS. We propose a modifed $\tau$ identification and a new evidence of signal in this study.

\section{Event generation and simulation}

Events have been generated with PYTHIA[4] event generator and effects of the CMS detector have been simulated using fast detector simulation package CMSJET[5]. Important event generation parameters are :

- $\hat{\mathrm{p}_{\mathrm{T}}}>20 \mathrm{GeV}$;

- Jets and leptons within $|\eta|<2.4$ have been considered;

- CTEQ2L parton distribution function[6] has been used.

We have assumed the initial low luminosity option $\left(10^{33} \mathrm{~cm}^{-2} \mathrm{~s}^{-1}\right)$ and have not included the effect of event pile up. Events have been generated for $\mathrm{M}_{\mathrm{H}^{+}}=\mathrm{M}_{\mathrm{W}}, 100,120,140$ and $160 \mathrm{GeV} / \mathrm{c}^{2}$ and for $\tan \beta=2,5,10,20,30$ and 40 as shown in Figure 1 . The $\mathrm{H}^{+}$is assumed to decay into $\tau^{+} \nu$ and $\mathrm{c} \overline{\mathrm{s}}$ only. Dependence of the branching ratio $\mathrm{BR}\left(\mathrm{t} \rightarrow \mathrm{bH}^{+}\right)$ on $\mathrm{M}_{\mathrm{H}^{+}}$and $\tan \beta$ is shown in Figure 2.

The polarisation state of $\tau$ coming from $W$ and charged Higgs decay affect the energy distribution of the decay products of $\tau[7]$. This has been taken care of by re-weighting the respective events. For $\mathrm{W}$ and $\mathrm{H}^{ \pm}$the effect goes in opposite directions: the energy spectrum of $\pi^{+}$from $\mathrm{H}^{+} \rightarrow \tau^{+} \nu_{\tau} ; \tau^{+} \rightarrow \pi^{+} \overline{\nu_{\tau}}$ is harder than those coming from $\mathrm{W}^{ \pm}$and

\footnotetext{
${ }^{1)}$ Unless otherwise stated, charge conjugate interactions are also implied.
} 


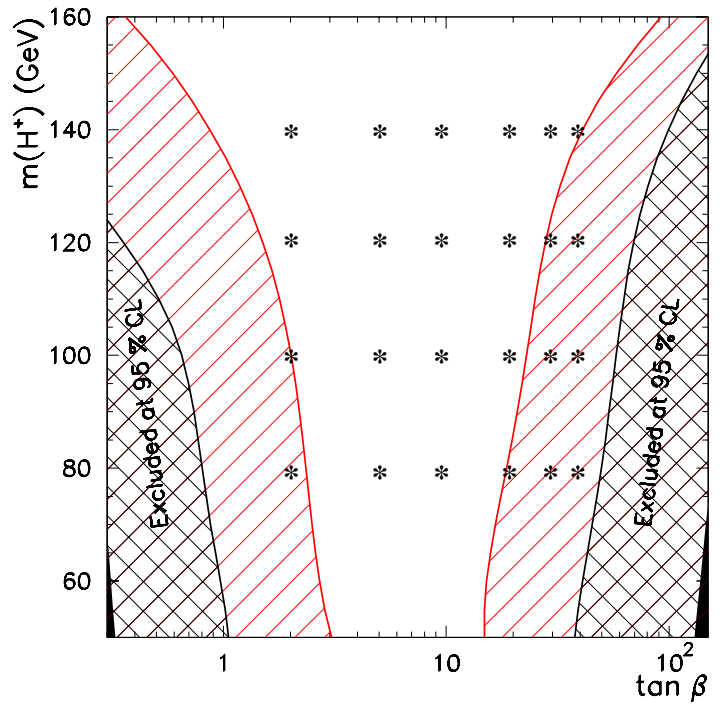

Figure 1: The area in the parameter space marked by cross is already excluded at 95\% CL by Tevatron RunI data and the hashed area should be excluded by RunII. The points shown correspond to generated event samples.

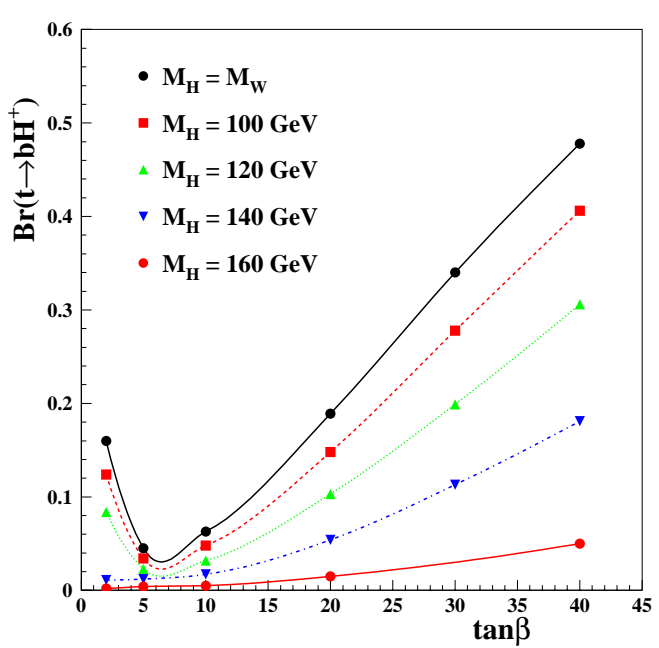

Figure 2: Dependence of the branching ratio $\mathrm{BR}\left(\mathrm{t} \rightarrow \mathrm{bH}^{+}\right)$on $\tan \beta$ for different $\mathrm{M}_{\mathrm{H}^{+}}$values.

largely cancel out in the total number of candidate events with $\tau$-jets in our analysis(see section 3.2 ).

The fast detector simulation package CMSJET simulates the calorimeter response. For charged particles it smears the momenta assuming $100 \%$ efficiency. Charged tracks and energy deposits in the calorimeter are combined to form jets using a cone algorithm[5]. The 'hits' with $\mathrm{E}_{\mathrm{T}}>1 \mathrm{GeV}$ are preselected and ordered in $\mathrm{E}_{\mathrm{T}}$. Hits are assigned to 'preclusters' $\left(\mathrm{E}_{\mathrm{T}}>10 \mathrm{GeV}\right)$ within a cone of radius of $\Delta \mathrm{R}=\sqrt{\Delta \eta^{2}+\Delta \phi^{2}}<0.5$.

\section{Analysis}

At LHC pp collisions will produce a large number of t $\mathrm{t}$ events $(\sigma(\mathrm{pp} \rightarrow \mathrm{t} \overline{\mathrm{t}}+\mathrm{X})=475 \mathrm{pb})$. We will also have a large number of single-top events $(\sigma(\mathrm{pp} \rightarrow \mathrm{t}+\mathrm{X})=170 \mathrm{pb})$. The branching fraction for $\mathrm{t} \rightarrow \mathrm{bH}^{+}$will depend on $\tan \beta$ and $\mathrm{M}_{\mathrm{H}^{+}}$(see figure 2). We look for events where one top decays semileptonically $\left(\mathrm{t} \rightarrow \mathrm{b} \ell^{+} \nu\right)$. This energetic lepton $(e, \mu)$ alongwith $\mathbb{E}_{\mathrm{T}}$ tag the $\mathrm{t} \overline{\mathrm{t}}$-events. Similar signals can come from $\mathrm{W}^{+} \mathrm{W}^{-}$events also.

\subsection{Preselection of $t \bar{t}$ events}

We preselect a sample rich in $t \bar{t}$ events. The criteria are :

- $\mathrm{N}_{\text {jet }} \geq 2$ each with $\mathrm{p}_{\mathrm{T}}^{\text {jet }}>20 \mathrm{GeV}$ and $\mathrm{E}^{\text {jet }}>40 \mathrm{GeV}$; 

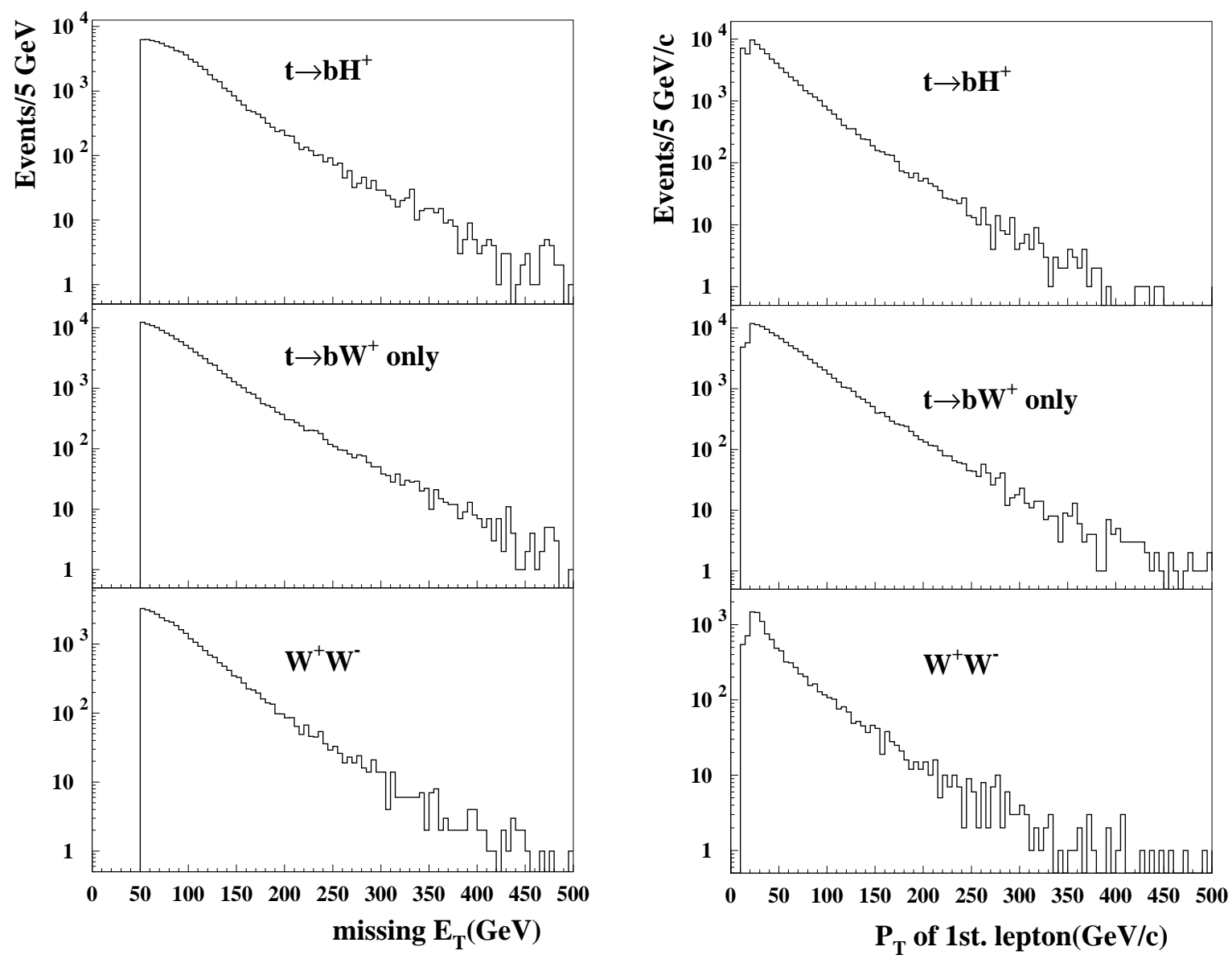

Figure 3: $\mathbb{E}_{\mathrm{T}}$ distribution of events in the Figure 4: $\mathrm{p}_{\mathrm{T}}$ distribution of the most enerpreselected sample(see text). getic lepton in the preselected sample.

- $\mathrm{N}_{\ell} \geq 1$ with $\mathrm{p}_{\mathrm{T}}^{\ell}>20 \mathrm{GeV}$;

- $\mathrm{E}_{\mathrm{T}} \geq 50 \mathrm{GeV}$

Figures 3 and 4 respectively show the spectra of missing energy and the $\mathrm{p}_{\mathrm{T}}$ of the most energetic lepton in signal and background events.

The efficiency of selecting t $\overline{\mathrm{t}}$ events varies between $16.8 \%$ and $15.5 \%$ depending on mass of $\mathrm{H}^{ \pm}$and $\tan \beta$. For events where top decays only to $\mathrm{bW}^{+}$the efficiency is $16.8 \%$. The purity of the $t \bar{t}$ sample is $\sim 93 \%$. The impurities are from single top events $(\sim 5.7 \%)$ and $\mathrm{W}^{+} \mathrm{W}^{-}$events $(\sim 1.6 \%)$. The jets and leptons in an event are ordered in decreasing $\mathrm{p}_{\mathrm{T}}$.

\subsection{Events with $\tau$-Jet}

In the preselected sample we look for events with at least one candidate $\tau$-jet :

$$
\mathrm{t} \overline{\mathrm{t}} \rightarrow \mathrm{bH}{ }^{+} \overline{\mathrm{b}} \mathrm{W}^{-} \rightarrow \mathrm{b} \tau^{+} \nu \overline{\mathrm{b}} \ell^{-} \bar{\nu}
$$



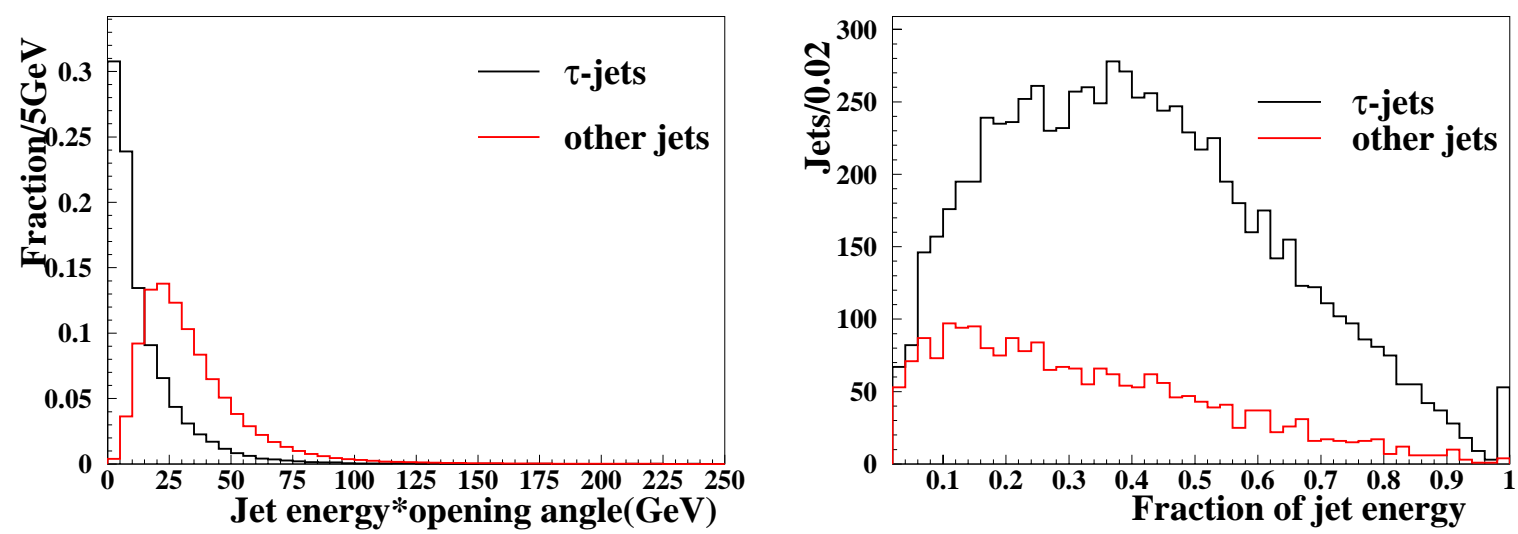

Figure 5: Discriminating variables for $\tau$-jet selection : product of jet opening angle and jet energy (left); fraction of jet energy carried by the hardest track in the jet (right), after the cut on the jet narrowness is applied.

where the $\tau$ decays hadronically. We identify $\tau$-jet as a narrow hadronic jet. Since the opening angle of the jet ${ }^{2)}$ will depend on the energy of the decaying $\tau$, we take product of the opening angle and the jet energy as the discriminating variable. Also, the jet has to have a charged track carrying a significant part of the jet energy. Figure 5 shows distributions for the product of jet energy and jet angle and for the fraction of jet energy carried by the most energetic track for $\tau$ and other jets. Based on these distributions, we demand that at least one of the first two jets satisfy the $\tau$ jet selection criteria :

- $\mathrm{E}_{\text {jet }} \cdot \theta_{\text {open }}^{\text {jet }} \leq 5 \mathrm{GeV}$

- $\mathrm{E}_{\text {trk }} / \mathrm{E}_{\mathrm{jet}}>0.3$

In the final selection we tighten the cut on missing energy : $\mathbb{E}_{\mathrm{T}}>60 \mathrm{GeV}$.

The rather stringent selection criteria leaves us with a small fraction of signal events. The number of candidate events show significant excess over the background for a nominal luminosity of $10 \mathrm{fb}^{-1}$. We see that for selected signal events(with at least one $\mathrm{t} \rightarrow \mathrm{bH}^{+}$ decay) $88-90 \%$ of the candidate $\tau$-jets are true $\tau$-jets. For $\mathrm{t} \overline{\mathrm{t}}$ events with $\mathrm{t} \rightarrow \mathrm{bW}^{+}$only the purity is $\sim 61 \%$. A small fraction of the selected event sample comes from single top events $(5.4 \%)$ and from $\mathrm{W}^{+} \mathrm{W}^{-}$events $(5.6 \%)$. These numbers have been estimated in the absence of signal and vary slightly in the presence of signal.

\subsection{Dilepton events}

An interesting aspect of presence of signal is the decrease of dilepton events in the sample passing the preliminary selection. The $\mathrm{p}_{\mathrm{T}}$ spectrum of the most energetic and the second most energetic lepton in dilepton event sample from top decaying through $\mathrm{bH}^{+}$and $\mathrm{bH}^{+}$

\footnotetext{
${ }^{2}$ The largest of the angles between hits in the jet and the jet direction is taken as the jet opening angle.
} 

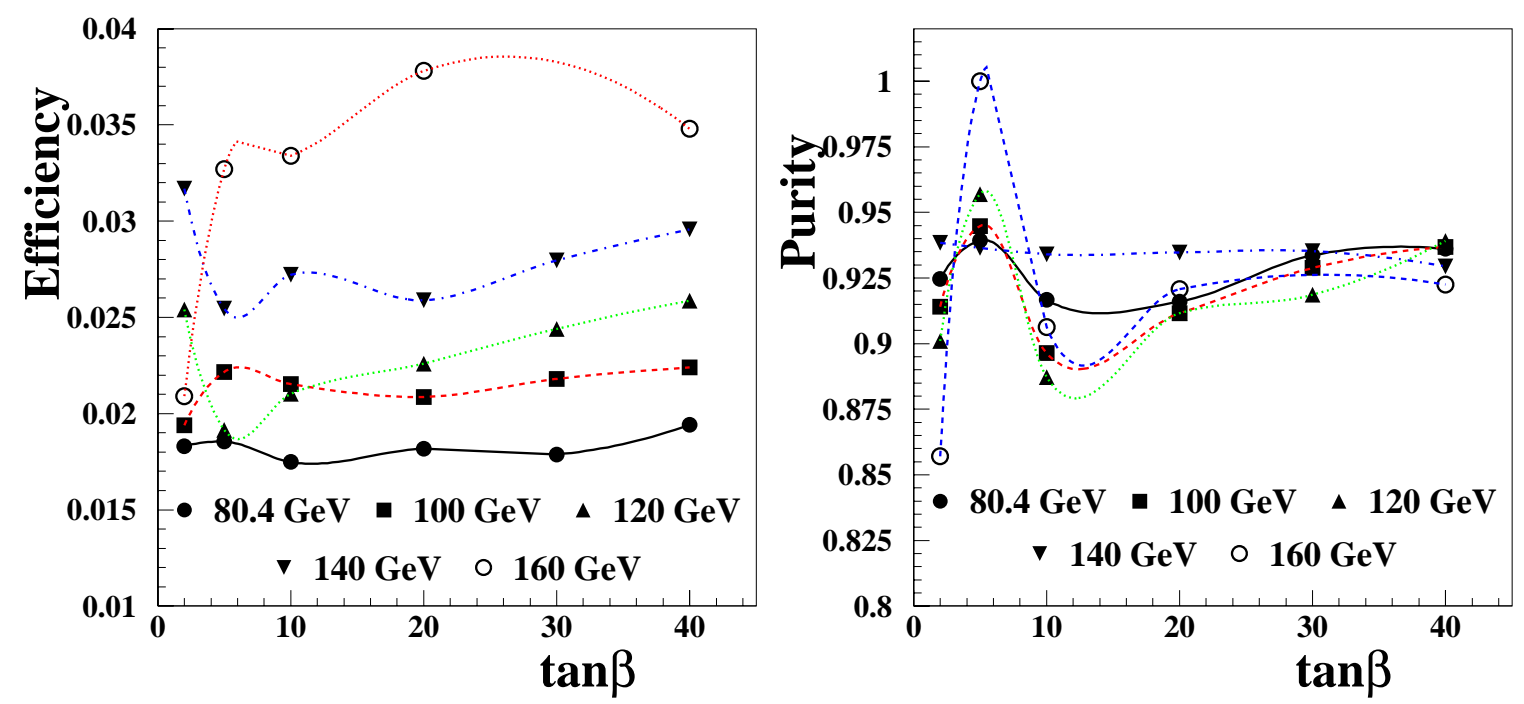

Figure 6: Efficiency of selecting signal events(see text) for different $\mathrm{M}_{\mathrm{H}^{+}}$is shown(left). Purity of the $\tau$-jets in the selected sample is also shown(right).

are shown in Figure 7. We demand $\mathrm{N}_{\ell} \geq 2$ and $\mathrm{p}_{\mathrm{T}}>20 \mathrm{GeV}$ for the first and second lepton. In the parameter space under consideration $\mathrm{H}^{+}$decays mostly into a $\tau^{+}$:

$$
\mathrm{t} \rightarrow \mathrm{bH}^{+} ; \mathrm{H}^{+} \rightarrow \tau^{+} \nu_{\tau} ; \tau^{+} \rightarrow \ell^{+} \overline{\nu_{\tau}} \nu_{\ell}
$$

Hence the leptons from $\mathrm{t} \rightarrow \mathrm{bH}^{+}$decay have lower energy compared to those from $\mathrm{t} \rightarrow$ $\mathrm{bW}^{+}, \mathrm{W}^{+} \rightarrow \ell^{+} \nu_{\ell}$ and are more likely to fail the $\mathrm{p}_{\mathrm{T}}$-cut. This deficit is an important confirmation of the presence of signal. It is interesting to note that except for a few events $(<0.1 \%)$ the leptons are of opposite sign, confirming that they are coming from $\mathrm{t} \rightarrow \mathrm{b} \ell^{+} \nu$ or $\mathrm{t} \rightarrow \mathrm{bH} H^{+} \rightarrow \mathrm{b} \tau^{+} \nu$ decays.

\section{Result}

We can exploit the above two different aspects of $\mathrm{H}^{ \pm}$decays for the search, namely excess of $\tau$ jets and depletion of dilepton events. Since there is no certain way of estimating the number of signal events in the selected event samples we have to look for excess (deficit) of $\tau$-jets $\left(\ell^{+} \ell^{-}\right)$over the expected sample size in the absence of signal events. We define the excess and significance as

$$
\mathrm{N}_{\tau}^{\text {excess }}=\mathrm{N}_{\tau}^{\text {sel }}-\mathrm{N}_{\tau}^{\mathrm{sel}, 0} ; \mathrm{S}_{\tau}=\frac{\mathrm{N}_{\tau}^{\text {excess }}}{\sqrt{\mathrm{N}_{\tau}^{\text {sel, }, 0}}}
$$

where $\mathrm{N}_{\tau}^{\text {sel, } 0}$ is the expected sample size with $\tau$-jet in the absence of signal. Similarly deficit and significance for $\ell^{+} \ell^{-}$sample is defined.

We estimate the luminosity required for exclusion of $\mathrm{t} \rightarrow \mathrm{bW}^{+}$only hypothesis(at $2 \sigma$ level) for both the signatures for each combination of $\mathrm{M}_{\mathrm{H}^{+}}$and $\tan \beta$. We take the lower one of the two as the required luminosity $\left(\mathcal{L}_{e x c l}\right)$. Similarly $\mathcal{L}_{d i s c}$ is determined for discovery 

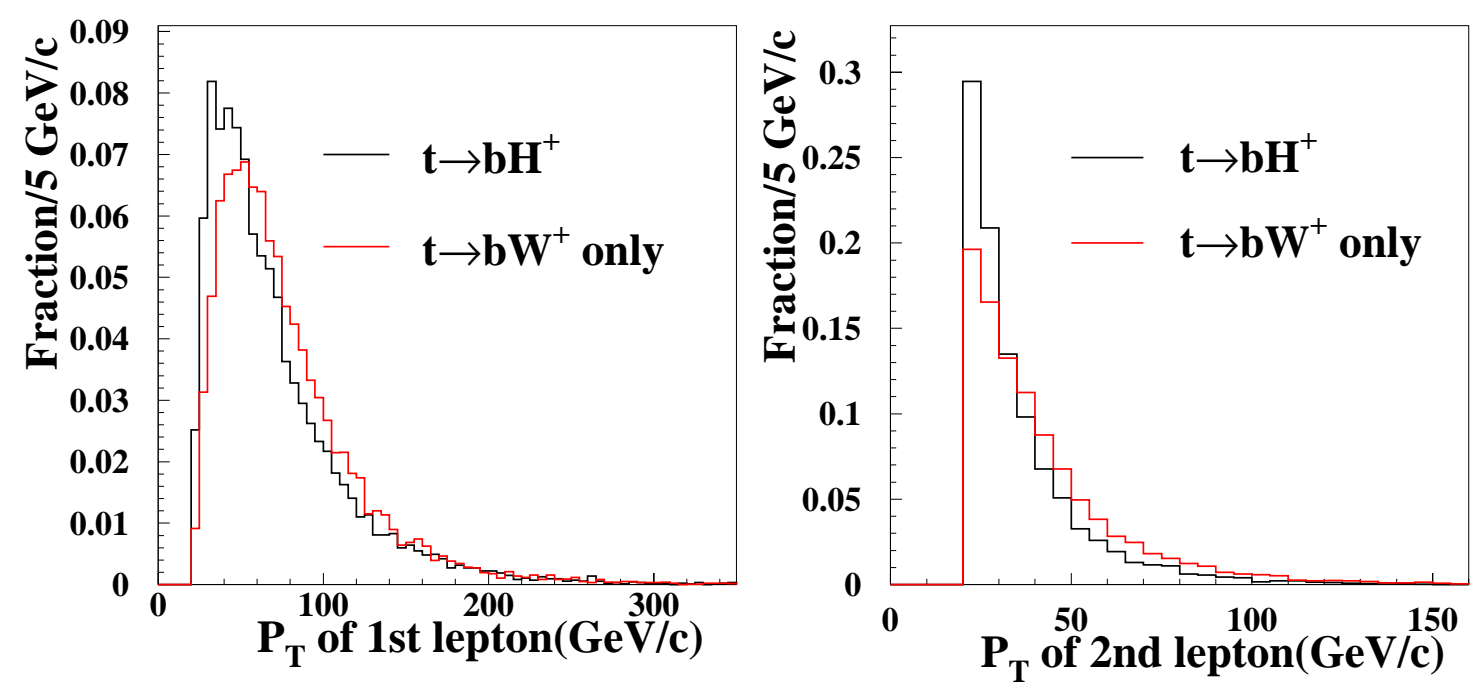

Figure 7: $\mathrm{p}_{\text {т }}$ spectrum of the first two leptons(ordered in decreasing $\mathrm{p}_{\mathrm{T}}$ ) in dilepton events in preselected $t \bar{t}$ sample. Signal events have $\mathrm{M}_{\mathrm{H}^{+}}=\mathrm{M}_{\mathrm{W}}$.

at $5 \sigma$ level. Figure 8 shows the $\mathcal{L}_{\text {excl }}($ left $)$ and $\mathcal{L}_{\text {disc }}$ (right) for different values of $\mathrm{M}_{\mathrm{H}^{+}}$. Since it is difficult to determine $\mathrm{M}_{\mathrm{H}^{+}}$or $\tan \beta$ it is important to estimate $\mathcal{L}_{\text {excl }}$ and $\mathcal{L}_{\text {disc }}$ for a given $\mathrm{M}_{\mathrm{H}^{+}}$over the range of $\tan \beta$. We see from figure 8 that for exclusion and discovery respectively $10 \mathrm{fb}^{-}$and $30 \mathrm{fb}^{-}$luminosities are necessary for $\mathrm{M}_{\mathrm{H}^{+}} \leq 160 \mathrm{GeV}$ for $2<\tan \beta<40$. Exclusion may be possible upto $170 \mathrm{GeV}$.
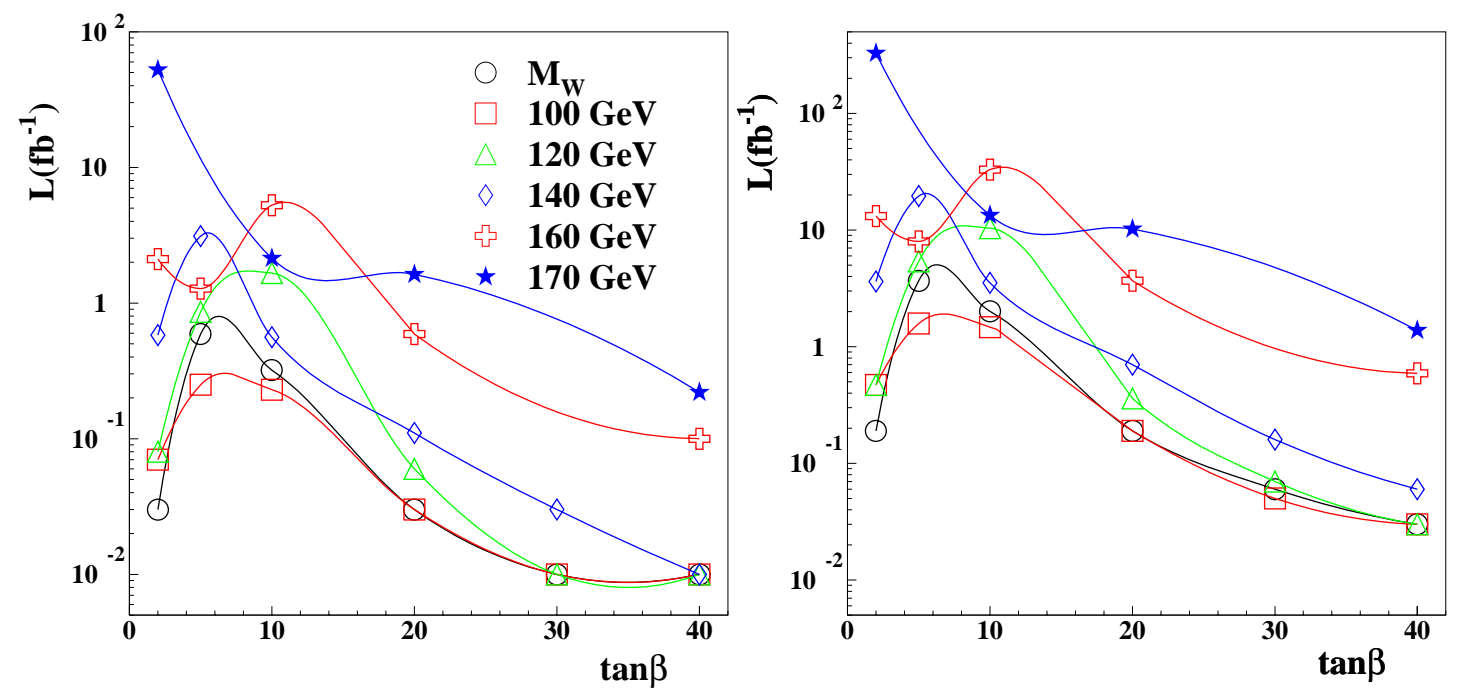

Figure 8: The minimum required luminosities(see text) for different $\mathrm{M}_{\mathrm{H}^{+}}$and $\tan \beta$ are shown : $\mathcal{L}_{\text {excl }}($ left $)$ and $\mathcal{L}_{\text {disc }}($ right $)$. 


\section{Conclusion}

We have proposed a new search strategy for charged Higgs at LHC : a modified $\tau$-selection

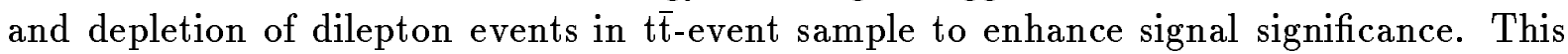
strategy is expected to exclude or discover $\mathrm{H}^{ \pm}$with mass up to $160 \mathrm{GeV}$ (for $2 \leq \tan \beta \leq$ 40 ) in the low luminosity run of LHC. Masses up to $170 \mathrm{GeV}$ may be excluded with the same data sample.

We are thankful to S. Raychaudhuri for valuable discussions regarding $\tau$ polarisation.

\section{References}

[1] ALEPH Collaboration, Phys. Lett. B450 467(1999); Contributed paper to EPS 99, EPS-HEP99 7-414;

DELPHI Collaboration, Phys. Lett. B460 484-497(1999); Contributed paper to Recontre de Moriond 2000, DELPHI 2000-029;

L3 Collaboration, Phys. Lett. B466 71-78(1999);

L3 Note 2510(March,2000)

Phys. Lett. B 446(1999) 368-377. OPAL Collaboration, Phys. Lett. B426 180192(1998); OPAL Physics Note PN421, Dec, 1999.

[2] CDF Collaboration, hep-ex/9912013;

DØ Collaboration, Phys. Rev. Lett. 82 4975-4980(1999).

[3] R. Kinnunen, J. Tuominiemi and D. Denegri, CMS TN/94-223.

[4] T. Sjostrand, Computer Physics Commun. 82 (1994) 74.

[5] S. Abdullin, A. Khanov and N. Stepanov; CMS TN-1994/180.

[6] James Botts, et al. Phys. Lett. B304 159-166, 1993.

[7] S. Raychaudhuri and D.P. Roy :

Phys. Rev. D52(1556-1564), 1995;

Phys. Rev. D53(4902-4908), 1996. 